\title{
Chest Exposure to X-Rays of Female Interventional Electrophysiologist
}

\author{
Giaccardi $M^{1 *}$, Turreni $F^{2}$, Rossi $F^{3}$, Chechi $T^{4}$ and \\ Mazzocchi $\mathbf{S}^{5}$ \\ ${ }^{1}$ Cardiology and Electrophysiology Unit, S. Maria Nuova \\ Hospital, Italy \\ ${ }^{2}$ Cardiology and Electrophysiology Unit, Belcolle \\ Hospital, Viterbo, Italy \\ ${ }^{3}$ Health Physics Unit Azienda Ospedaliera Universitaria \\ Careggi, Italy \\ ${ }^{4}$ Cardiology Unit, S. Maria Annunziata Hospital, Italy \\ ${ }^{5}$ Health Physics Unit Azienda USL Toscana Centro, \\ Florence Empoli, Italy \\ *Corresponding author: Marzia Giaccardi, \\ Department of Internal Medicine, Electrophysiology Unit, \\ Santa Maria Nuova Hospital, P.zza Santa Maria Nuova, \\ 150122, Firenze, Italy
}

Received: January 20, 2021; Accepted: February 10 2021; Published: February 17, 2021

\begin{abstract}
Interventional Female Cardiologists (WIC) operating in high case mix laboratories are exposed to a significant chest X-Ray scattered dose from the patient. In this setting stochastic effect may be highly detrimental because of breast radiation sensitivity. Aim of this study is to measure and optimize WICs' chest radiation exposure in a high case mix electrophysiology laboratory, in order to validate and implement the use of personal protective equipment and lead equivalent glass viewing window, and to evaluate chest X-Ray exposure behind the protective equipment.
\end{abstract}

Keywords: Breast cancer; X-Ray exposure; Lead apron; Protective equipment

\section{Abbreviations}

WIC: Woman Interventional Cardiologist; BC: Breast Cancer; IR: Ionizing Radiation; IC: Interventional Cardiologists; LB: Left Breast; LUA: Left Upper Abdomen

\section{Introduction}

Breast Cancer (BC) is the second term in total world malignancy incidence ranking, and the most common malignant disease in women [1]. The genetic influence on BC susceptibility in general population is $27 \%$, while environmental and lifestyle factors show a primary role in carcinogenesis [2]. Among these, Ionising Radiation (IR) exposure has been clearly associated to increased risk of female $\mathrm{BC}$ in several populations, including atomic bomb survivors, medically exposed populations and occupationally exposed cohorts [3]. Among the latter category, Interventional Cardiologists (IC) are the most exposed amongst $\mathrm{x}$-ray using personnel [4]. Recent advances within this discipline have led to the treatment of more complex diseases with subsequent lengthy procedures and higher radiation exposure [5]. Indeed, cardiologists working in high-volume catheterisation laboratories have higher levels of somatic DNA damage when compared with matched clinical cardiologists [6] and have an elevated risk of $\mathrm{BC}$ and melanoma due to daily low dose radiation exposure over several years $[7,8]$. Females are $38 \%$ more sensitive to malignant damage for any given radiation exposure, and breast tissue is the most likely developed cancer $[9,10]$. Subsequently, personal protection with radioprotective equipment is a standard measure in any interventional setting. Standard lead apron may be empowered by one or two additional sleeves in addition to dedicated breast shields [11]. In order to evaluate the actual chest protection by means of this additional personal protective equipment, we quantified total thorax X-Ray dose exposure in a first operator Interventional Female Cardiologist (WIC) wearing sleeves empowered lead apron in a high case mix electrophysiology laboratory, eventually highlighting possible additional protection devices.

\section{Materials and Methods}

Between March and August 2020, we quantified the IR exposure of a single first operator WIC after further $\mathrm{X}$ ray protection optimization in the laboratory layout and in the lead apron protection system. In order to evaluate the consequent thoracic IR incident dose and to verify the impact of varying operational and environmental conditions, we manufactured an under-the-lead-apron wearable cotton jacket, with a fixed position sewed set of 60 dosimeters (Figure 1a). Interdosimeter distance varied from $5 \mathrm{~cm}$ to $2.5 \mathrm{~cm}$ depending on the thorax region to examine. Dosimeters 1-5 monitored data related to left upper thorax, 6-21 to left breast, 22-32 to left upper abdomen, 33-36 to right upper thorax, 37-50 to right breast and 51-60 to upper abdomen region (Figure 1b). The used dosimeters are plastic-covered, single-crystal detectors, TLD100 type thermoluminescent crystals, in the Ext-Rad configuration, manufactured by Harshaw Thermofisher. During the study period TLD dosimeters were replaced bimonthly. The first interval ranged from March to April, the second from May to June, the third from July to August 2020. After replacement Dosimeters were processed with an Harshaw 6600P automatic reader by the accredited service in compliance with the ISO 17025 standard [12]. Dosimeters are type tested, according to IEC 62387 standard [13]. Responses were quantified in terms of air kerma. Because of Covid 19 pandemic and subsequent hospital emergency needs, Electrophysiology laboratory moved from the usual room and setting, during the first and second aforementioned periods, in a previous operating room which -given the provisional nature of the set-up in relation to the emergency- was not suspended ceiling shielding equipped. The $\mathrm{X}$ ray system used in this setting was a Philips Pulsera mobile c-arm X-ray unit. In the last period (July-August), the EP laboratory returned to the usual room and setting, provided with
Austin J Womens Health - Volume 8 Issue 1 - 2021

Submit your Manuscript | www.austinpublishing group.com

Giaccardi et al. (c) All rights are reserved 


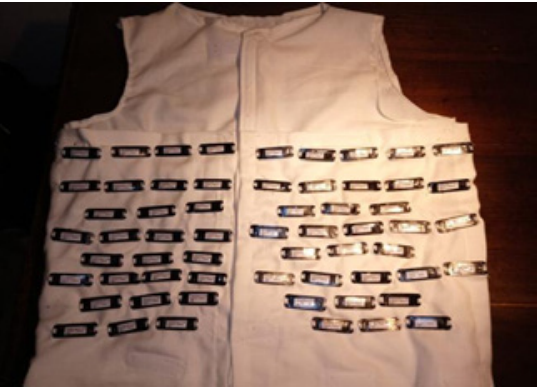

Figure 1a: Under-the-lead-apron wearable cotton jacket with fixed position sewed set of 60 TLD-100 thermoluminescence dosimeters.

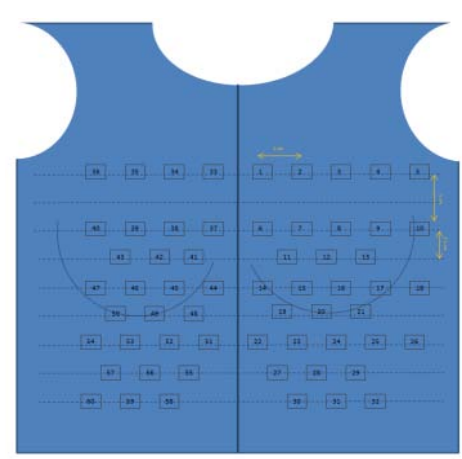

Figure 1b: recording dosimeters position: 1-5 Left Upper Thorax (LUT), 6-21 Left Breast (LB), 22-32 to Left Upper Abdomen (LUA), 33-36 to Right Upper Thorax (RUT), 37-50 to Right Breast (RB) and 51-60 to Right Upper Abdomen region (RUA).

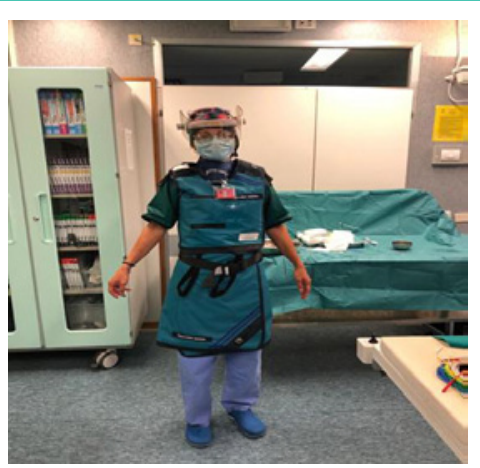

Figure 2: Axillary-Shoulder Sleeves Empowered Wearable anti-X protective equipment for a first operator WIC.

suspended ceiling lead shield and operating table suspended lead drape. The used angiography system was a Eurocolumbus Euroampli Alien Cardio. The WIC is equipped with an anti-X protective apron consisting of a 'lead free' fully enveloping jacket and skirt, with a certified equivalent protection level of $0.5 \mathrm{mmPb}$ in front and of $0.25 \mathrm{mmPb}$ in back. The protective equipment has been tested before the use. The size is optimized for the body of the operator, and the armholes adhere optimally to the axillary cavity. Moreover, the WIC wears two lead equivalent sleeves covering the shoulders and axillary cavities to protect maximally from the scatter radiations, even during complex and extremely patient-close procedures. First operator also wears lead glasses with lateral protection or lead equivalent face shield, for the lenses of the eyes protection (Figure 2). Natural background

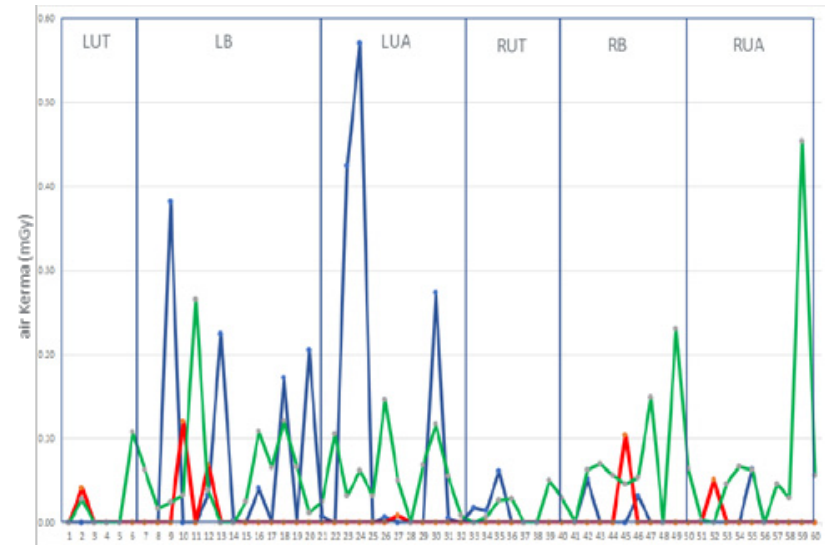

Figure 3: Cumulative torso dose during the observational study. Ordinata: measured air kerma values ( $m G v$ ). Abscissa: fixed dosimeter number. From left to right plotted dose data related to: LUT (dosimeter 1-5), LB (6-21), LUA (22-32), RUT (33-36), RB (37-50), RUA (51-60). Color code: Blue: first period; Red: second period; Green: third period. Abbreviations as in Figure $1 \mathrm{~b}$.

radiation was subtracted using unexposed dosimeters, annealed and analysed together with the others. The detection limit was $0,06 \mathrm{mGy}$. Measurement uncertainty is about $20 \%$ with a coverage factor $\mathrm{k}=2$. A table with the procedures performed in each period and the patient dose indicators have been filled by the cardiologist: KAP, air kerma area product, and fluoroscopy time in each procedure.

\section{Results}

Dosimeter sewed jacket and personal empowered protective equipment were correctly worn during all procedures. Body dose quantification at the end of the observation period despite the proper use of personal and environmental protective equipment is show in Figure 3. Despite the low workload during the initial Covid pandemic, total body exposition was higher in the first observational period than in the following. This data is related to the first period during which the laboratory was initially moved to a previous operating room not provided by ceiling moving shields and with a different $\mathrm{X}$-ray interventional system. The second period cumulative dose is lower despite a similar number and complexity of the procedures. In the first two periods, urgent procedures only were carried out, like complete AV block, or secondary prevention pacemaker and ICD implantation respectively. Four CRT implant only were performed, three of which in the first period. In the third period, upon returning to the usual electrophysiology room, equipped with additional IR shields and 3D non fluoroscopic approach, usual electrophysiological activity restarted with a slower than normal increase, because of the lack of non-COVID dedicated hospital beds. In this period, 33 procedures were performed with fluoroscopic exposure and 27 with zero fluoroscopy approach. In Table 1 the number of performed procedures, patient KAP and fluoroscopy time mean values and

Table 1: KAP patient radiation exposure indicator and fluoroscopy time (mean values and deviation standard for procedure).

\begin{tabular}{|c|c|c|c|}
\hline Period & $\begin{array}{c}\text { N. of fluoroscopic } \\
\text { procedures }\end{array}$ & KAP $\left(\mathrm{Gy} \mathrm{cm}^{2}\right)$ & Fluoroscopy time (min) \\
\hline First & 6 & $30 \pm 27$ & $12 \pm 9,5$ \\
\hline Second & 7 & $33 \pm 65$ & $5 \pm 1,7$ \\
\hline Third & 33 & $24 \pm 31$ & $6,5 \pm 6,3$ \\
\hline
\end{tabular}


standard deviations are reported for the three monitored time periods. The majority of dose values in samples points are below the lowest level of detection $(<0.06 \mathrm{mGy})$, and up to $0.57 \mathrm{mGy}$ in the abdomen region even in the first period (blue line data). The WIC's body areas consistently mostly exposed to scatter radiation were the Left Breast (LB) and the Left Upper Abdomen (LUA) in concordance with previous evidence [23], even in the second and third periods. The evaluation of each single procedure showed that the radiological exposure peaks were related to more complex procedures such as CRT implants (data not shown), which imply closer proximity to the patient and greater use of lateral radiological projections. The use of suspended ceiling lead shield and suspended operating table lead drape, present in the electrophysiology room in Cardiology in the third period further lowered the cumulative dose despite the higher procedural number and complexity, especially if compared to the first period.

\section{Discussion}

Radiation related damage risk awareness is a prerequisite to create and implement the culture of respect for radiation hazard, a commitment to minimise exposure and maximise protection, mostly in high-risk personnel, i.e. IC [14]. Industry has developed innovative solutions to reduce by several folds the IR dose per examination in all fields of medical imaging, from cardiac CT to nuclear perfusion imaging, up to near zero fluoroscopy in electrophysiology [1517] nevertheless this may not be sufficient. Despite an increasing proportion of women in most fields of medicine, the majority of IC is still mostly represented by males. One of the causes for this might be the previous interpretation of data on radiation exposure as a harmful effect on fertility and pregnancy, although previous data suggest that appropriate precautions may stochastically reduce related risk [18]. Indeed, concern about the harmful effects of X-Ray guided interventional activity is warranted. Previous data highlight orthopaedic illness, cataract, skin lesions, and cancer significantly increased incidence in IC when compared with matched unexposed subjects, as with longer duration of occupational work [19]. In female radiation exposed personnel particular attention should be paid to breast tissue exposure, because of intrinsic radiation and cumulative exposure vulnerability, leading to linearly increased dose related $\mathrm{BC}$ risk [20-22]. Previous data show that IC Body radiation exposure is not homogeneous, being more evident in the upper thorax portion, highlighting the upper outer breast quadrant as the most common site of BC incidence [23]. Therefore, every effort must be pursued to reduce IR exposure in this area, especially in female interventional operators. For these reasons we tested the hypothesis of reduced IR exposure in this area by means of sleeves empowered aprons and tested monitored different environmental factors affecting it. Our data show that IR over the chest of a first operator WIC is not neglectable but reduced to a very low dose by the use of personal empowered protective equipment, and that additional environmental measures and tools can further lower the cumulative dose, i.e. the use of fluoroscopy and acquisition optimized protocols, 3D non fluoroscopic systems, additional ceiling fixed X shields and operating table lead drapes. In these conditions IR exposure is reduced to an acceptable level, considering that IR risk is stochastically zeroed by exposition avoidance only. We confirm that left breast and left upper abdomen are the most exposed sides. We therefore recommend the following strategies as standards to limit intraoperative radiation exposure and to potentially reduce the risk of $\mathrm{BC}$ in WIC who performs many procedures: use sleeves empowered, properly sized and fitted, lead aprons for breast protection; implement as possible zero fluoroscopy $3 \mathrm{D}$ navigation systems approach or, when $\mathrm{X}$ rays are necessary, reduce IR dose by minimising frame rate and time, the acquired cine images and non-digital zoom use; avoid highscattering projection exposure (steep caudal and cranial angulation); increase as possible the distance between the WIC and the $\mathrm{x}$-ray source, especially during lateral/oblique views; increase as possible the distance between the $\mathrm{x}$-ray source and the patient in order to decrease scatter radiation $[11,24]$ : this may be particularly important in procedures such as CRT implantation or complex ablation, which may imply proximity to the patient and use of lateral radiological projections; use a quality performance control assurance program for imaging equipment; routinely wear personal dosimeters to follow radiation exposure among personnel $[25,26]$; protect the eye lens by means of XRay glasses [27].

In this setting of our active research and previous literature, the Zero-Gravity suspended radiation protection system and the radiation protection cabin are further shielding systems, designed to increase the level of radiation protection enabling the operators to work without wearing the lead apron nor any additional protective equipment, with negligible radiation exposure for the operators $[28,29]$. Furthermore, the cardiologist's awareness of the meaning of the patient dose indicators is important: the greater the KAP value for the patient, the greater the radiation diffused to the team.

\section{Conclusion}

For a first operator, high case mix WIC it is possible to achieve nearly negligible effective dose values in terms of probability of all known radiation effects during a full professional life pursuing optimised conditions. X ray equipment periodical quality control, empowered personal protective devices, environmental proper technique utilisation seem to be protective in high workload and complex procedures electrophysiology laboratories. Moreover, the cardiologist, and mostly an WIC should be aware of the IR exposition risk in order to properly use all the abovementioned radiation protection systems. The TLD dosimeter jacket continuously monitored the incident IR dose at the operator's breast level and highlighted the possible need for further optimisation in exposure reduction, i.e. improvement in the use of lead ceiling shields, the use of sterile $\mathrm{X}$ ray attenuating drapes on the patient, in addition to dedicated breast shields [30].

\section{References}

1. Parkin DM. Global cancer statistics in the year 2000. Lancet Oncol. 2001; 2: $533-543$

2. Lichtenstein P, Holm NV, Verkasalo PK, Iliadou A, Kaprio J, Koskenvuo M, et al. Environmental and heritable factors in the causation of cancer - analyses of cohorts of twins from Sweden, Denmark, and Finland. N Engl J Med. 2000; 343: 78-85.

3. Ronckers CM, Erdmann CA, Land CE. Radiation and breast cancer: a review of current evidence. Breast Cancer Res. 2005; 7: 21-32.

4. Venneri L, Rossi F, Botto N, Andreassi MG, Salcone N, Emad A, et al. Cancer risk from professional exposure in staff working in cardiac catheterization laboratory: insights from the National Research Council's Biological Effects of Ionizing Radiation VII Report. Am Heart J. 2009; 157: 118-124. 
5. Picano E, Vañò E, Rehani MM, Cuocolo A, Mont L, Bodi V, et al. The appropriate and justified use of medical radiation in cardiovascular imaging: a position document of the ESC Associations of Cardiovascular Imaging Percutaneous Cardiovascular Interventions and Electrophysiology. European Heart Journal. 2014; 35: 665-672.

6. Andreassi MG, Cioppa A, Botto N, Joksic G, Manfredi S, Federici C, et al. Somatic DNA damage in interventional cardiologists: A case-control study. FASEB J. 2005; 19: 998-999.

7. Rajaraman P, Doody MM, Yu CL, Preston DL, Miller JS, Sigurdson A, et al. Cancer risks in US radiologic technologists working with fluoroscopically guided interventional procedures, 1994-2008. Am J Roentgenol. 2016; 206: 1101-1108.

8. Ho TL, Shieh SH, Lin CL, Shen WC, Kao CH. Risk of cancer among cardiologists who frequently perform percutaneous coronary interventions: a population-based study. Eur J Clin Invest. 2016; 46: 527-534.

9. Committee to Assess Health Risks from Exposure to Low Levels of Ionizing Radiation, National Research Council. Health risks from exposure to low levels of ionizing radiation: Beir VII phase 2. The National Academies Press. 2006.

10. Boice JD, Mandel JS, Doody MM. Breast cancer among radiologic technologists. JAMA. 1995; 274: 394-401.

11. Buchanan GL, Chieffo A, Mehilli J, Mikhail GW, Mauri F, Presbitero P, et al. Women In Innovation Group. The occupational effects of interventional cardiology: results from the WIN for Safety survey. Eurolntervention. 2012; 8: 658-663.

12. General requirements for the competence of testing and calibration laboratories. EN ISO/IEC 17025:2017.

13. Radiation protection instrumentation-Dosimetry systems with integrating passive detectors for individual, workplace and environmental monitoring of photon and beta radiation. IEC 62387. 2020

14. Giaccardi M, Anselmino M, Del Greco M, Mascia G, Paoletti Perini A, Mascia $P$, et al. Radiation awareness in an Italian multispecialist sample assessed with a web-based survey. Acta Cardiologica. 2020; 31: 1-5.

15. Thaker A, Navadeh S, Gonzales H, Malekinejad M. Effectiveness of policies on reducing exposure to ionizing radiation from medical imaging: a systematic review. J Am Coll Radiol. 2015; 12: 1434-1445.

16. Anselmino M, Sillano D, Casolati D, Ferraris F, Scaglione M, Gaita F. A new electrophysiology era: zero fluoroscopy. J Cardiovasc Med. 2013; 14 221-227.

17. Giaccardi M, Del Rosso A, Guarnaccia V, Ballo P, Mascia G, Chiodi L, et al. Nearzero $x$-ray in arrhythmia ablation using a 3-dimensional electroanatomic mapping system: a multicenter experience. Heart Rhythm. 2016; 13: 150156.

18. Yong CM, Abnousi F, Rzeszut AK, Douglas PS, Harrington RA, Mehran
$\mathrm{R}$, et al. Sex Differences in the Pursuit of Interventional Cardiology as a Subspecialty Among Cardiovascular Fellows-in-Training. JACC. 2019; 11: 219-228.

19. Andreassi MG, Piccaluga E, Guagliumi G, Del Greco M, Gaita F, Picano E. Occupational Health Risks in Cardiac Catheterization Laboratory Workers. Circ Cardiovasc Interv. 2016; 9: e003273.

20. Boice JD, Mandel JS, Doody MM. Breast cancer among radiologic technologists. JAMA. 1995; 274: 394-401.

21. Doody MM, Freedman DM, Alexander BH, Hauptmann M, Miller JS, Rao RS, et al. Breast cancer incidence in U.S. Radiologic technologists. Cancer. 2006; 106: $2707-2715$

22. Ronckers CM, Erdmann CA, Land CE. Radiation and breast cancer: a review of current evidence. Breast Cancer Res. 2005; 7: 21-32.

23. Kwong S. Laterality, detailed site, and histology of female breast cancer, California, 1988-1999. In: Morris CR, Kwong SL, editors. Breast cancer in California. 2003.

24. Valone LC, Chambers M, Lattanza L, James MA. Breast Radiation Exposure in Female Orthopaedic Surgeons. J Bone Joint Surg Am. 2016; 98: 18081813.

25. Hirshfeld JW, Ferrari VA, Bengel FM, Bergersen L, Chambers CE, Einstein AJ, et al. 2018 ACC/HRS/NASCI/SCAI/SCCT Expert Consensus Document on Optimal Use of Ionizing Radiation in Cardiovascular Imaging-Best Practices for Safety and Effectiveness, Part 2: Radiological Equipment Operation, Dose-Sparing Methodologies, Patient and Medical Personnel Protection. J Am Coll Cardiol. 2018; 71: 2829-2855.

26. Durán A, Hian SK, Miller DL, Heron JL, Padovani R, Vano E. A summary of recommendations for occupational radiation protection in interventional cardiology. Catheterization and Cardiovascular Interventions. 2012; 81: 562-567.

27. Early and late effects of radiation in normal tissues and organs: threshold doses for tissue reactions and other non-cancer effects of radiation in a radiation protection context. Annals of the ICRP.Ref 4844-6029-7736. 2011.

28. Dragusin O, Weerasooriya R, Jaïs P, Hocini M, Ector J, Takahashi J, et al. Evaluation of a radiation protection cabin for invasive electrophysiological procedures. Eur Heart J. 2007; 28: 183-189.

29. Haussen DC, Van Der Bom IMJ, Nogueira RG. A prospective case control comparison of the ZeroGravity system versus a standard lead apron as radiation protection strategy in neuroendovascular procedures. J Neurointerv Surg. 2016; 8: 1052-1055

30. Vlastra W, Delewi R, Sjauw KD, Beijk Bimmer MA, Claessen E, Streekstra $\mathrm{G}$, et al. Efficacy of the RADPAD Protection Drape in Reducing Operators' Radiation Exposure in the Catheterization Laboratory Circulation. Circ Cardiovasc Interv. 2017; 10: e006058. 